Textures and Microstructures, 1987, Vol. 7, pp. 29-72

Photocopying permitted by license only

(C) Gordon and Breach Science Publishers Inc., 1987

Printed in the United Kingdom

\title{
Calculation of the Yield Locus of Textured Polycrystals Using the Taylor and the Relaxed Taylor Theory
}

\section{P. VAN HOUTTE}

Department of Metallurgy and Materials Engineering, Katholieke Universiteit Leuven, de Croylaan 2 B-3030 Leuven, Belgium

(Received 18 April 1986)

Several authors have proposed methods which use the Taylor-Bishop-Hill theory in order to calculate the yield surface of textured samples of which the O.D.F. is known.

The purpose of this paper is to show how these methods can be generalized while keeping the computational effort within reasonable limits. It must be emphasized that the new method produces "true" plane sections of the yield locus instead of so-called "principle strain yield loci."

A theorem that permits the exploitation of the sample symmetry is demonstrated. After a general description of the method, it is explained how the theorem can be used in order to restrict the number of deformation modes that must be considered.

The next section discusses how a data bank of Taylor factors can be constructed. The full-constraint Taylor theory as well as the relaxed Taylor theory are considered.

In the next section, it is explained how the plane sections through the multidimensional yield locus are generated. A few applications are finally discussed, including a study of the elongation of a torsion sample of which the O.D.F. has been measured.

\section{INTRODUCTION}

The plastic anisotropy of a material is often described by a yield surface. It is the geometrical representation of the stress states for which plastic yielding occurs. Such stress state is described by a tensor $\mathbf{S}$, represented by a matrix $S_{i j}$ for a given reference system in 
physical space. The tensor $\mathbf{S}$ is symmetric, thus

$$
S_{i j}=S_{j i}
$$

Hence there are only 6 independent components $S_{i j}$. A six dimensional space is sufficient to represent a yield surface. An analytical representation would be:

$$
F\left(S_{i j}\right)=\text { constant }
$$

For isotropic materials, plastic yielding only depends on the value of the principal stresses, not on the principal stress directions. This means that the yield locus can be represented in a three dimensional principal stress space. This simplification is however impossible for anisotropic materials. For materials, for which plastic yielding is independent of the hydrostatic component $S^{\prime \prime}$ of the stress the number of dimensions can nevertheless be reduced to five:

$$
S^{\prime \prime}=\left(S_{i i}\right) / 3
$$

This allows to replace the stress $\mathbf{S}$ by the deviatoric stress $\mathbf{S}^{\prime}$ :

$$
S_{i j}^{\prime}=S_{i j}-\delta_{i j} S^{\prime \prime}
$$

for which the following property always holds

$$
S_{i i}^{\prime}=0
$$

There are only five independent deviatoric stress components. Materials for which plastic yieldings is independent from the hydrostatic component of the stress do not change their volume during plastic deformation. This is expressed by

$$
\dot{E}_{i i}=0
$$

In which $\dot{E}_{i j}$ are the components of the plastic strain rate tensor $\dot{\mathbf{E}}$. We will consider Eq. (6) as valid throughout this paper.

The number of mechanical tests required to measure a five dimensional yield locus is tremendous. Therefore, it is often assumed that the anisotropy is only caused by the crystallographic anisotropy of the sample. It is then theoretically derived using crystal plasticity laws. This is not so difficult for a single crystal. For 
a polycrystal, two things are required:

i) the measurement of the crystallite orientation distribution function (O.D.F.);

ii) a reliable model for the plastic yielding of a polycrystal.

In this paper, we will consider the O.D.F. measured by means of the serial expansion method (Bunge, 1982). The Taylor-BishopHill theory will be chosen as model for polycrystal plasticity (Gil Sevillano, Van Houtte and Aernoudt, 1980). This means, that the resulting yield curves are only valid for offsets that are sufficiently large to reach multiple slip in every grain (a few percent). The elasto-plastic transition range cannot be studied.

Various authors have already proposed such methods (Bunge, 1970; Parniere and Roesch, 1975; Grumbach, Parniere, Roesch and Sauzay, 1975; Bunge, 1979; Bunge, Schulze and Grzesik, 1980; Sowerby, Da Viana and Davies, 1980). They did not intend to construct a complete yield locus in five dimensional stress space, but limited the analysis to a plane section of it, showing a yield curve as a function of $S_{11}^{t}$ and $S_{22}^{t}$. These are the 11 and 22 components of the stress tensor in the $\left[x_{1}^{t}, x_{2}^{t}, x_{3}^{t}\right]$ reference system of a tensile test sample, cut from a rolled sheet which itself has a $\left[x_{1}, x_{2}, x_{3}\right]$ reference system (Figure 1).

The analytical description of the section plane is given by:

$$
S_{23}^{t}=S_{31}^{t}=S_{12}^{t}=0
$$

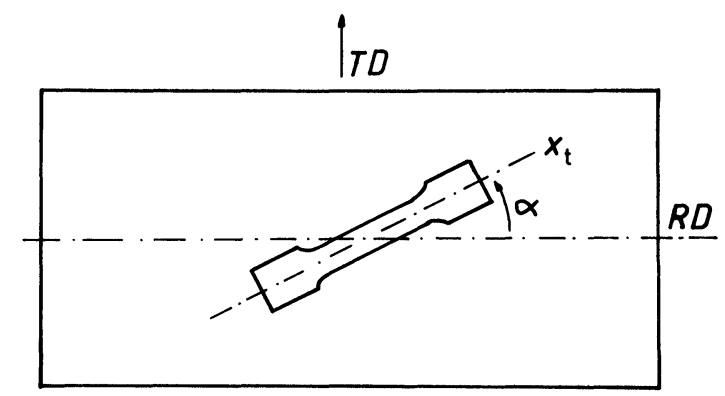

FIGURE 1 Tensile test sample cut from a rolled sheet. Reference system of the sheet: $x_{1}=$ rolling direction (RD); $x_{2}=$ transverse direction (TD); $x_{3}=$ sheet plane normal (SPN). Reference system of the test sample: $x_{1}^{t}=$ tensile axis $\left(x^{t}\right) ; x_{3}^{t}=x_{3}$ and $x_{2}^{t}=$ transverse direction of the sample (perpendicular to $x_{1}^{t}$ and $x_{3}^{t}$ ). 
The implementation of this condition is not easy, as will be demonstrated below. In previous work (Canova, Kocks, Tomé and Jonas, 1985; Bunge et al., 1980; Sowerby et al., 1980), it was replaced by a much easier condition:

$$
\dot{E}_{23}^{t}=\dot{E}_{31}^{t}=\dot{E}_{12}^{t}=0
$$

$x_{1}, x_{2}$ and $x_{3}$ usually are twofold symmetry axes of the texture of a rolled sheet. It can be demonstrated (see below) that in that case Eq. (7) and Eq. (8) are equivalent for $\alpha=0$ and $\alpha=\pi / 2$ but not for other values of $\alpha$ (the angle between the rolling direction and the tensile axis-Figure 1). The physical meaning of this is, that for angles other than 0 or $\pi / 2$, the plastic response of the anisotropic material to a uniaxial tensile stress may be a plastic strain for which $x_{t}$ is not a direction of principal strain, i.e. $\dot{E}_{12}^{t}$ may be different from zero. Models which neglect this fact by using Eqs. (8) instead of Eqs. (7) do not produce true yield loci but so-called "principal strain yield loci".

The method proposed by Mols, Van Praet and Van Houtte (1984) really uses Eqs. (7) and produces a polyhedron-shaped envelope of the five dimensional yield surface. It is stored in computer memory under the form of the equations of 51408 hyperplanes (the facets of the polyhedron). The computer can then at will produce any plane section, for example those that correspond to $S_{11}^{t}-S_{22}^{t}$ sections for any value of $\alpha$, but also $S_{12}^{t}-S_{32}^{t}$ sections. The method is suited for cubic metals without Bauschinger effect. Monoclinic and orthorhombic sample symmetries can be treated. A serious drawback is the amount of calculations that must be performed, which prevents interactive exploitation of the yield surface using a graphical terminal.

A simplified version of this method is presented in this paper. The amount of calculations has been reduced 30 times, which makes an interactive exploitation possible. One additional assumption was necessary: at least one principal direction of the stress should coincide with one twofold symmetry axis of the texture of the sample (sample symmetry, not crystal symmetry, as explained by Bunge (1982)). Nearly all practical applications known to the author can nevertheless be treated by this less-general method.

It must be pointed out that there are other ways as well to improve the classical methods for yield locus prediction. Berveiller 
and Zaoui (1984) for example proposed to replace the TaylorBishop-Hill theory by an elasto-plastic self-consistent method, which would give more precise results for small offset yield loci.

\section{THEOREM}

\subsection{Introduction}

The simplification that is presented in this paper is an application of a theorem concerning the symmetry properties of anisotropic materials.

Suppose that an anisotropic sample possesses a twofold rotation axis of symmetry, at least with regard to those material properties that control plastic anisotropy. Then this axis will be a principal direction of strain when it is a principal direction of stress.

A few examples will illustrate the principle of the twofold rotation symmetry axis of the sample. The simplest assumption (also adopted in the present paper) is, that the anisotropy only depends on the crystallographic texture. The twofold rotation symmetry axis that is meant here belongs to the sample symmetry of the texture, not to the crystal symmetry (see e.g. Bunge (1982)). Figure 2 shows two pole figures of a cold rolled sheet of an aluminium alloy.

The sheet plane normal (SPN) is represented at the centre of the
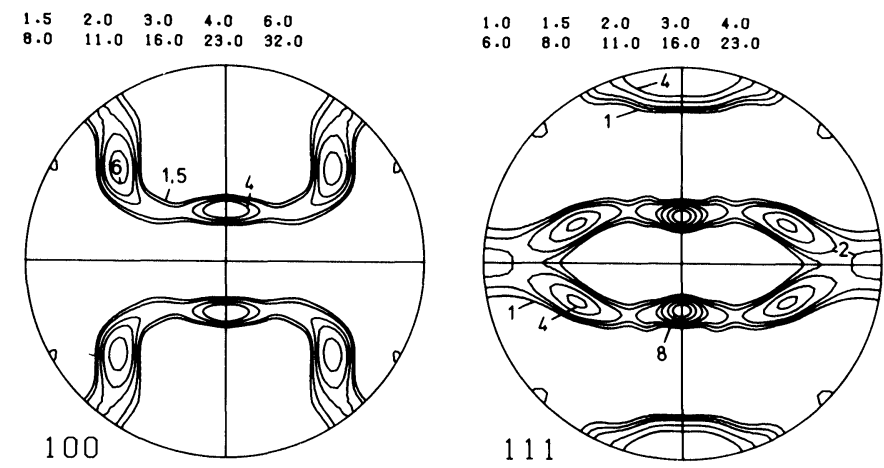

FIGURE $2\{111\}$ and $\{200\}$ pole figures of the texture of a $95 \%$ cold rolled aluminium alloy. 


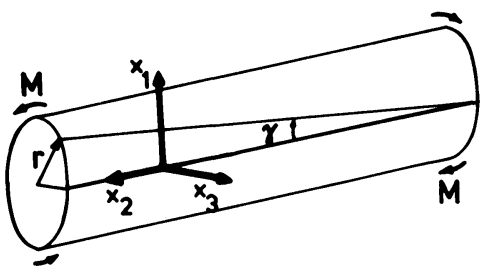

FIGURE 3 Choice of the reference system of the specimen used for pole figure measurements on a torsion test sample. $x_{1}$ is the shear direction, $x_{2}$ the shear plane normal and $x_{3}$ the radial direction.

pole figures. It is seen that a rotation $\pi$ around SPN transforms both pole figures into themselves. So the SPN is a twofold symmetry axis of these pole figures. In this example, the property would not only hold for these two pole figures, but for all pole figure of this sample. The SPN hence qualifies as a twofold symmetry axis to which the theorem applies. The rolling direction and the transverse direction would qualify as well.

Figure 3 shows a schematic representation of a torsion test sample. After torsion, the properties of such sample are no longer homogeneous. Consider an infinitely small piece of material, at the surface of the sample. A $\left(x_{1}, x_{2}, x_{3}\right)$ reference system is placed on it in Figure 3.

Figure 4 shows two pole figures of the resulting texture in the case of a copper sample. The $x_{3}$ axis (radial direction) is at the centre of
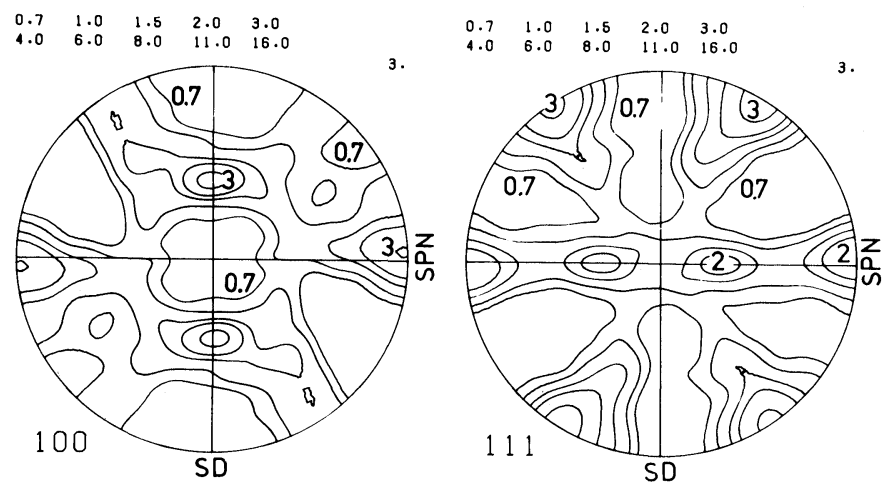

FIGURE 4 Pole figures of a copper-type torsion texture (Van Houtte et al., 1981). 
these pole figures. It is a twofold rotation axis of them and also of all other pole figures that can be made of this texture. It qualifies as a twofold rotation axis to which the theorem applies, at least for that tiny part of material, at the surface of the sample, of which the texture was measured. Note that the radial direction is a direction of principal strain and stress during the torsional deformation.

\subsection{Note concerning the implementation of the "sample symmetry" in a three-dimensional description of a crystallographic texture}

Pole figures such as those represented by Fig. 2 have "symmetry axes": the two-dimensional figure is transformed into itself by a reflection with respect to these axes. The latter then are often thought to be the intersections of "mirror planes" of the threedimensional texture with the plane of the figure. This extrapolation is incorrect: a "mirror plane" cannot be used to define a symmetry relation of a three-dimensional texture, although the concept has often been used, even in the same context as here (Canova et al., 1985).

A description of a three-dimensional texture essentially is a set of crystallite orientations, together with a corresponding set of volume fractions. An orthogonal reference system is put on the crystal lattice of each crystallite. For cubic metals, it is possible to adopt a convention that requires these to be right-turning reference systems. This simplifying convention is not always possible (Esling et al., 1980). Three Euler angles then describe the orientation of such reference system with respect to the external reference system of the sample. Roughly speaking, an operation (defined within the set of crystallite orientations) which relates an arbitrary crystal orientation $g_{A}$ to a crystal orientation $g_{B}$ is a "sample-symmetry operation" when the total volume of crystals with orientation $g_{A}$ is equal to the total volume of crystals with orientation $g_{B}$. It is implicitly assumed, that $g_{A}$ and $g_{B}$ are both elements of the set of orientations i.e. they must both describe the orientation of a right-turning reference system with respect to the sample reference system. A separate definition of special Euler angles would be required if one wanted to describe the orientation of a left-turning crystal reference system.

Symmetry operators in physical space are normally used to define 
symmetry operators in orientation space. A twofold rotation axis qualifies for this: one takes the reference system of crystal A (which is an object in physical space), rotates it over angle $\pi$ around the twofold rotation axis and one gets another valid crystal reference system of which the orientation $g_{B}$ can be calculated. On the other hand, a reflection in a mirror plane (performed in physical space) does not qualify. It would transform the right-turning reference system of crystal A into a left-turning reference system that is put on the lattice of crystal B. No Euler angles exist for this left-turning reference system. Note that a rotation by an angle $\pi$ around the normal of the mirror plane would also put the crystal reference system of crystal A on the lattice of crystal $B$, hence offering a valid alternative to the forbidden reflection.

\subsection{Proof of the theorem}

The theorem is proved by negative demonstration. Let $\left\langle x_{1}, x_{2}, x_{3}\right\rangle$ be a fixed reference system. $\left\langle x_{1}^{t}, x_{2}^{t}, x_{3}^{t}\right\rangle$ is a reference system fixed to the sample. Initially, they coincide. Let $x_{3}=x_{3}^{t}$ then be the twofold symmetry axis of the sample. Consider now that the sample is loaded and plastically deformed in such way, that $x_{3}=x_{3}^{t}$ is a principal direction of stress. This means that:

$$
S_{23}=S_{13}=0
$$

This can for example be achieved by a uniaxial tensile test in the direction $x_{3}$. Two different thought experiments are now carried out on the same sample:

1) A. the sample is loaded; plastic deformation takes place B. the deformed sample is rotated by an angle $\pi$ around $x_{3}$.

2) A. the sample is rotated by an angle $\pi$ around $x_{3}$, and so is the loading mechanism.

B. the sample is loaded; plastic deformation takes place.

The net result of both experiments must be identical. Now look what happens when during plastic deformation, $x_{3}=x_{3}^{t}$ would not be a principal direction of strain. Assume for example that there would be a non-zero shear $\dot{E}_{31}$, caused by a simple shear along the $O x_{2} x_{3}$ plane in the $O x_{1}$ direction. 


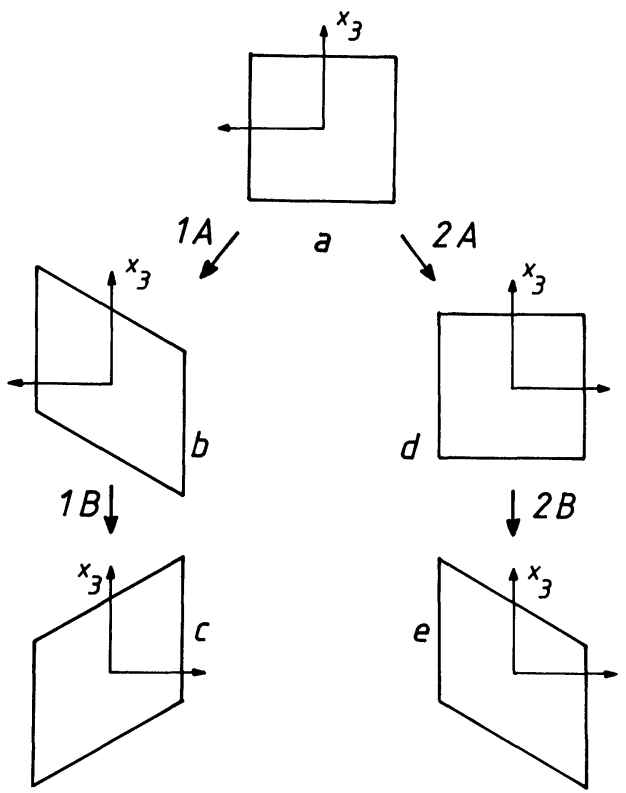

FIGURE 5 Two thought experiments.

Figure 5a shows the initial shape of the sample.

Figure $5 \mathrm{~b}$ shows the sample after experiment $1 \mathrm{~A}$.

Figure 5c shows the result after thought experiment $1 \mathrm{~B}$.

Figure 5d gives the sample after thought experiment $2 \mathrm{~A}$.

Now note that the configuration of Figure $5 \mathrm{~d}$ is physically exactly equivalent to the configuration of Figure 5a, because $x_{3}$ is a twofold symmetry axis of the sample, and at the same time a principal direction of stress. The operation (2B) is physically totally equivalent to the thought experiment (1A) since, because of Eq. (9), the stress tensor (expressed in the fixed reference system $\left\langle x_{1}, x_{2}, x_{3}\right\rangle$ remains unchanged after a rotation of the loading mechanism by an angle $\pi$ around $x_{3}$.

So the loading operations (1A) and (2B) are physically equivalent and act upon physically equivalent samples (5a and $5 \mathrm{~d})$. Hence the result must also be the same. For this reason, Figure 5e is a copy of Figure 5b. 
This then leads to an absurdity: Figure $5 \mathrm{c}$ and $5 \mathrm{e}$ are not identical, as they should be. Hence the assumption that $x_{3}=x_{3}^{t}$ is not a principal direction of the strain must be incorrect. The same idea can also be expressed as follows: when $x_{3}$ simultaneously is a twofold rotation symmetry axes of the sample and of the loading mode, then it must remain so during and after plastic deformation.

\section{DESCRIPTION OF THE METHOD}

The classical method of calculating a yield surface of a textured polycrystal consists of finding a set of hyperplanes which are tangent to it (Bunge et al., 1980; Sowerby et al., 1980; Mols et al., 1984; Canova et al., 1985). Each of these hyperplanes correspond to a certain strain mode, described by a strain rate tensor $\dot{E}_{i j}$. Let $g$ be short-hand rotation for a crystal orientation (Bunge, 1982). The Taylor-Bishop-Hill theory (Gil Sevillano et al., 1980) or a relaxed version of it (Van Houtte, 1984), can calculate the Taylor factor $M$ for a crystal with orientation $g$ (with respect to the specimen reference system $x_{1}, x_{2}, x_{3}$ ). Let $\dot{\gamma}_{s}$ be the rate of slip on slip system $s$. The Taylor factor then means:

$$
M=\left(\sum_{s=1}^{n}\left|\dot{\gamma}_{s}\right|\right) / \dot{E}_{e q}
$$

$\dot{E}_{e q}$ is the equivalent strain rate (of the macroscopic strain) as defined by some convention. The von Mises convention would be:

$$
\dot{E}_{e q}=\left((2 / 3) \dot{E}_{i j} \dot{E}_{i j}\right)^{1 / 2}
$$

It can then be shown (Gil Sevillano et al., 1980) that for that crystal, the plastic work rate per unit volume is given by:

$$
\dot{W}=\tau^{\mathrm{c}} M \dot{E}_{e q}
$$

in which $\tau^{\mathrm{c}}$ is the critical resolved shear stress of the metal crystal (assumed to be equal on all slip systems). Equation (12) is then averaged on the whole polycrystal:

$$
\dot{W}=\tau^{\mathrm{c}} \bar{M} \dot{E}_{e q}
$$


with

$$
\bar{M}=\oint M(g) f(g) d g
$$

The average Taylor factor $\bar{M}$ no longer is a function of the crystal orientation $g$, but still is one of the strain mode $\dot{E}_{i j}$. $f(g)$ is the crystal orientation distribution function of the textured metal, which must be measured (Bunge, 1982). The averaging procedure (Eq. (14)) is made easier when the series expansion method is used. Assume that, for a given strain mode $\dot{E}_{i j}$, a Taylor-Bishop-Hill computer code or a relaxed version of it was used to obtain the $M$ values for all possible crystal orientations $g$, and that these data were used to obtain the series expansion of $M$ itself (it will be explained below how this work can be organized):

$$
M(g) \approx \sum_{l}^{L} \sum_{\mu}^{N_{1}(l)} \sum_{v}^{N_{2}(l)} m_{l}^{\mu v} \dot{\bar{T}}_{l}^{\mu v}(g)
$$

in which $\tilde{T}_{l}^{\mu v}(g)$ are symmetrical generalized spherical harmonics of order $l, \mu, v$ (see Bunge, 1982). $m_{l}^{\mu \nu}$ are the corresponding coefficients. A set of such coefficients describes a function $M(g)$ with a certain precision, which depends on the degree $L$ of the series expansion. The coefficients $m_{l}^{\mu v}$ do not depend on the texture. So several sets of them (for several deformation modes $\dot{E}_{i j}$ ) can be stored on disk or on tape once and for all.

The averaging procedure Eq. (14) now becomes:

$$
\bar{M}=\sum_{l} \sum_{\mu} \sum_{v} \frac{m_{l}^{\mu v} C_{l}^{\mu v}}{2 l+1}
$$

in which $C_{l}^{\mu \nu}$ are the series expansion coefficients of the texture. They must be measured. For $L=22$, there are 124 such coefficients of even rank $l$ and 61 of odd rank $l$, together 185 . So the evaluation of expression (16) roughly requires 185 multiplications and 185 additions. The required computer time is very small.

The average work rate per unit volume is given by another expression:

$$
\bar{W}=\dot{E}_{i j} S_{i j}
$$

So it follows from Eq. (13) that

$$
\dot{E}_{i j} S_{i j}=\tau^{\mathrm{c}} \bar{M} \dot{E}_{e q}
$$




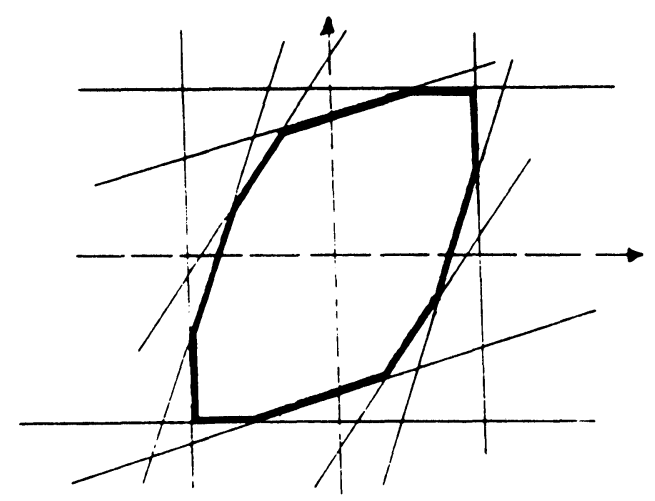

FIGURE 6 The yield locus is tangent to all hyperplanes of the type of Eq. (18). An envelope to the yield curve can thus be obtained in a plane section of stress space.

For a given strain mode $\dot{E}_{i j}, \bar{M}$ can be obtained using Eq. (16). Eq. (18) then becomes a linear equation for the unknown stress components $S_{i j}$. In stress space, it represents a hyperplane that somewhere contains one point of the yield locus. So an envelope to the yield surface is obtained if a lot of these hyperplanes are constructed (Figure 6).

Note that Eq. (18) is independent of the convention for $\dot{E}_{\text {eq }}$. Indeed: by using Eq. (10), it can be written as:

$$
\dot{E}_{i j} S_{i j}=\tau^{\mathrm{c}} \sum_{s=1}^{n}\left|\dot{\gamma}_{s}\right|
$$

in which $\dot{E}_{e q}$ is completely absent.

\section{GENERATION OF DEFORMATION MODES AND CALCULATION OF THE CORRESPONDING M-VALUES}

\subsection{Introduction}

Fig. 6 shows how to estimate the yield locus from a set of hyperplanes. Each hyperplane corresponds to a deformation mode, characterized by a strain rate tensor $E_{i j}$. The magnitude of the strain rate is unimportant; only the ratio of one component to another 
matters. This tensor $\dot{E}_{i j}$ is symmetric:

$$
\dot{E}_{i j}=\dot{E}_{j i}
$$

Because of condition (6), it has only five independent components. This means that a tremendous number of distinct strain rate tensors must be considered when trying to cover all possible deformation modes within a certain angular resolution. Such a method has nevertheless been developed (Mols et al., 1984). After using it for some time, it was concluded that

i) the textures of the considered samples nearly all had at least a twofold symmetry axis;

ii) nearly all stress states that were of some practical interest, and for which plane sections of the yield locus had to be made, were such that this twofold symmetry axis also was a principal direction of stress.

These two condiserations led to the idea, that the number of deformation modes that must be considered could perhaps be reduced strongly by limiting the method to stress states for which a twofold symmetry axis of the sample would be a principal direction of stress. The theorem that has been explained in section 2 permits to see how this stress restriction also restricts the strain modes that must be considered.

Let $x_{3}$ be a principal direction of stress. This means that

$$
S_{23}=S_{13}=0
$$

(cfr. Eq. (9)).

According to the theorem the $x_{3}$ axis also is a principal direction of the plastic strain mode that corresponds to such stress state. Hence:

$$
\dot{E}_{23}=\dot{E}_{13}=0
$$

for the plastic strain rate tensor. Let $\left\langle x_{1}^{P}, x_{2}^{P}, x_{3}^{P}\right\rangle$ be a reference system that coincides with the principal strain axes.

Since $x_{3}=x_{3}^{P}$, only one angle $\psi$ suffices to characterize the orientation of this reference system with respect to the macroscopic reference system of the sample $\left\langle x_{1}, x_{2}, x_{3}\right\rangle$ (Figure 7).

In the reference system $\left\langle x_{1}^{P} x_{2}^{P} x_{3}^{P}\right\rangle$ the strain rate tensor can be 




FIGURE $7\left\langle x_{1}, x_{2}, x_{3}\right\rangle$ are the macroscopic reference axes of the sample. $x_{3}$ is a twofold symmetry axis [of the sample]. $\left\langle x_{1}^{P}, x_{2}^{P}, x_{3}^{P}\right\rangle$ is a reference system that is placed upon the principal directions of strain.

expressed as follows:

$$
\dot{E}_{i j}^{P}=\left[\begin{array}{ccc}
\dot{E}_{11}^{P} & 0 & 0 \\
0 & \dot{E}_{22}^{P} & 0 \\
0 & 0 & \dot{E}_{33}^{P}
\end{array}\right]
$$

The three components $\dot{E}_{11}^{P}, \dot{E}_{22}^{P}$ and $\dot{E}_{33}^{P}$ and the angle $\Psi$ are sufficient to characterize a deformation mode that satisfies condition Eq. (21). This means that all possible deformation modes can be generated by selecting appropriate values for these parameters. The number of deformation modes to be considered must of course be finite in practical calculation. It depends on the desired angular resolution in $\Psi$ and in principal strain space.

\subsection{Generation of the deformation modes}

Range for $\Psi$. We will let the angle $\Psi$ (Figure 7) vary from 0 to $\pi$ in steps of $\pi / 18\left(10^{\circ}\right)$. Note that the range from $\pi$ to $2 \pi$ would produce identical strain rate tensors.

In case of orthorhombic sample symmetry, this range can be reduced even more, at least for computational purposes (see Appendix I). 


\subsection{Choice of $\dot{E}_{11}^{P}, \dot{E}_{22}^{P}, \dot{E}_{33}^{P}$}

The selection of $\dot{E}_{11}^{P}, \dot{E}_{22}^{P}, \dot{E}_{33}^{P}$ is done in three ranges, each characterized by a parameter: $q_{1}, q_{2}, q_{3}$.

a) for $0 \leqslant q_{1} \leqslant 0.5$

$$
\begin{aligned}
& \dot{E}_{11}^{P}=\dot{E} \\
& \dot{E}_{22}^{P}=-q_{1} \dot{E} \\
& \dot{E}_{33}^{P}=-\left(1-q_{1}\right) \dot{E}
\end{aligned}
$$

$q_{1}$ corresponds to the "contraction ratio $q$ " defined by Bunge (1970).

b) for $0 \leqslant q_{2} \leqslant 0.5$ :

c) for $0 \leqslant q_{3} \leqslant 0.5$ :

$$
\begin{aligned}
& \dot{E}_{11}^{P}=\dot{E} \\
& \dot{E}_{22}^{P}=-\left(1-q_{2}\right) \dot{E} \\
& \dot{E}_{33}^{P}=-q_{2} \dot{E}
\end{aligned}
$$

$$
\begin{aligned}
& \dot{E}_{11}^{P}=-q_{3} \dot{E} \\
& \dot{E}_{22}^{P}=-\left(1-q_{3}\right) \dot{E} \\
& \dot{E}_{33}^{P}=\dot{E}
\end{aligned}
$$

The parameters $q_{i}$ take the values $0,0.1,0.2,0.29,0.37,0.44$ and 0.5 . These values correspond to equidistant steps in principal strain space. The value of $\dot{E}$ is unimportant, except that a distinction must be made between positive and negative values for materials that have asymmetric crystallographic deformation mechanisms such as mechanical twinning systems or $\{112\}\langle 111\rangle$ slip systems in b.c.c. metals with different $\tau^{\mathrm{c}}$ in positive and negative slip directions.

These three ranges together do not cover all possible strain rate tensors of the type given by Eq. (23). But the strain rate tensors that should be produced for the "missing" ranges at an angle $\Psi$ are identical to certain tensors already produced in the ranges above at an angle $\Psi+\pi / 2$. For example, the range

$$
0 \leqslant q_{4} \leqslant 0.5 \quad \begin{aligned}
& \dot{E}_{11}^{p}=-q_{4} \dot{E} \\
& \\
& \dot{E}_{22}^{P}=\dot{E} \\
& \\
& \dot{E}_{33}^{P}=-\left(1-q_{1}\right) \dot{E}
\end{aligned}
$$


would produce the same strain rate tensors as the range for $q_{1}$ (Eqs. (24-26)) at $\Psi+\pi / 2$.

So for each value of $\Psi, 21$ hyperplanes are generated. This makes a total of 399 hyperplanes, since 19 distinct values of $\Psi$ are considered.

This supposes positive values for $\dot{E}$ (Eqs. 24-32). To each of the 399 hyperplanes, there is another one for which the signs of the $\dot{E}_{i j}$ are reversed in Eq. (18), while conserving the positive sign of $\bar{M}$. The value of $\bar{M}$ is not affected, unless there is asymmetry in the crystallographic deformation mechanisms. This finally leads to $\mathbf{7 9 8}$ different hyperplanes. However, for samples with orthorhombic sample symmetry and with symmetric slip systems, the $\bar{M}$-values only need to be calculated for 210 of these hyperplanes: 21 deformations modes for 10 values of $\Psi$ between 0 and $\pi / 2$. The 588 other hyperplanes are then obtained from these by inversion of the strain rate tensor (see above) or by symmetry operations (see appendix I).

Expression for $\dot{E}_{i j}$. Let the tensor $R_{i j}$ express the transformation law from the $\left\langle x_{i}\right\rangle$ system to the $\left\langle x_{i}^{P}\right\rangle$ system (Fig. 7):

$$
R_{i j}=\left[\begin{array}{ccc}
\cos \Psi & \sin \Psi & 0 \\
-\sin \Psi & \cos \Psi & 0 \\
0 & 0 & 1
\end{array}\right]
$$

Equation (23) and Eqs. (24-32) give the strain rate tensor as expressed in the $\left\langle x_{i}^{P}\right\rangle$ system. Its expression in the reference system of the sample $\left\langle x_{i}\right\rangle$ is given by

$$
\dot{E}_{i j}=R_{k i} \dot{E}_{k l}^{P} R_{l j}
$$

\section{CALCULATION OF THE $\bar{M}$-VAlUes}

\subsection{Introduction}

The basic philosophy of the calculation of the $\bar{M}$-values is:

i) a data bank is constructed which in principle contains the 
$M$-value for all crystallite orientations in Euler space and for all deformation modes that are considered.

ii) The average $\bar{M}$-values of a polycrystal with a crystallographic texture are calculated using Eq. (14).

The practical implementation will now be discussed. For reasons of simplicity, only the case of orthorhombic sample symmetry and symmetric slip systems is explained. That means that the $\bar{M}$-values need only be calculated for 210 deformation modes (see the previous sections). The method can very easily be adapted to treat the monoclinic case (e.g. torsion texture) or the case that the deformation mechanisms are asymmetric, e.g. when mechanical twinning is present.

The $M$-values which are needed for the data bank can be constructed by means of a Taylor-Bishop-Hill model or by means of the relaxed Taylor-Bishop-Hill theory, for metals with flat elongated grains (Van Houtte, 1984b).

Figure 8a shows a schematic representation of such flat grain. In the classical Taylor-Bishop-Hill theory, thereafter called fullconstraint theory (FC), it is assumed that the strain rate tensors are identical for all grains. This requires the simultaneous activation of at least five slip systems.

In the relaxed constraints (RC) Taylor-Bishop-Hill theory, this condition is somewhat "relaxed."

In the "lath" version of the theory, the $\dot{E}_{13}$ component is left free: it need not be equal for all crystallites (Figure $8 \mathrm{~b}$ ). Only four slip systems need to be active. Finally, the $\dot{E}_{13}$ and the $\dot{E}_{23}$ components are both free in the "pancake" version of the theory (Figure 8c) (Van Houtte, 1984b).

The use of these models for the construction of a data bank of $M$-values will be discussed in the following order: (i) "lath" model, (ii) "pancake" model; (iii) FC model.

\subsection{Using the "lath" model}

The $M$-values must be calculated for all crystal orientation with respect of the sample reference systems $\left\langle x_{i}\right\rangle$. In practice, the $M$-values are only calculated for the nodal points of a grid in Euler space. The following grid could for example be used for cubic 

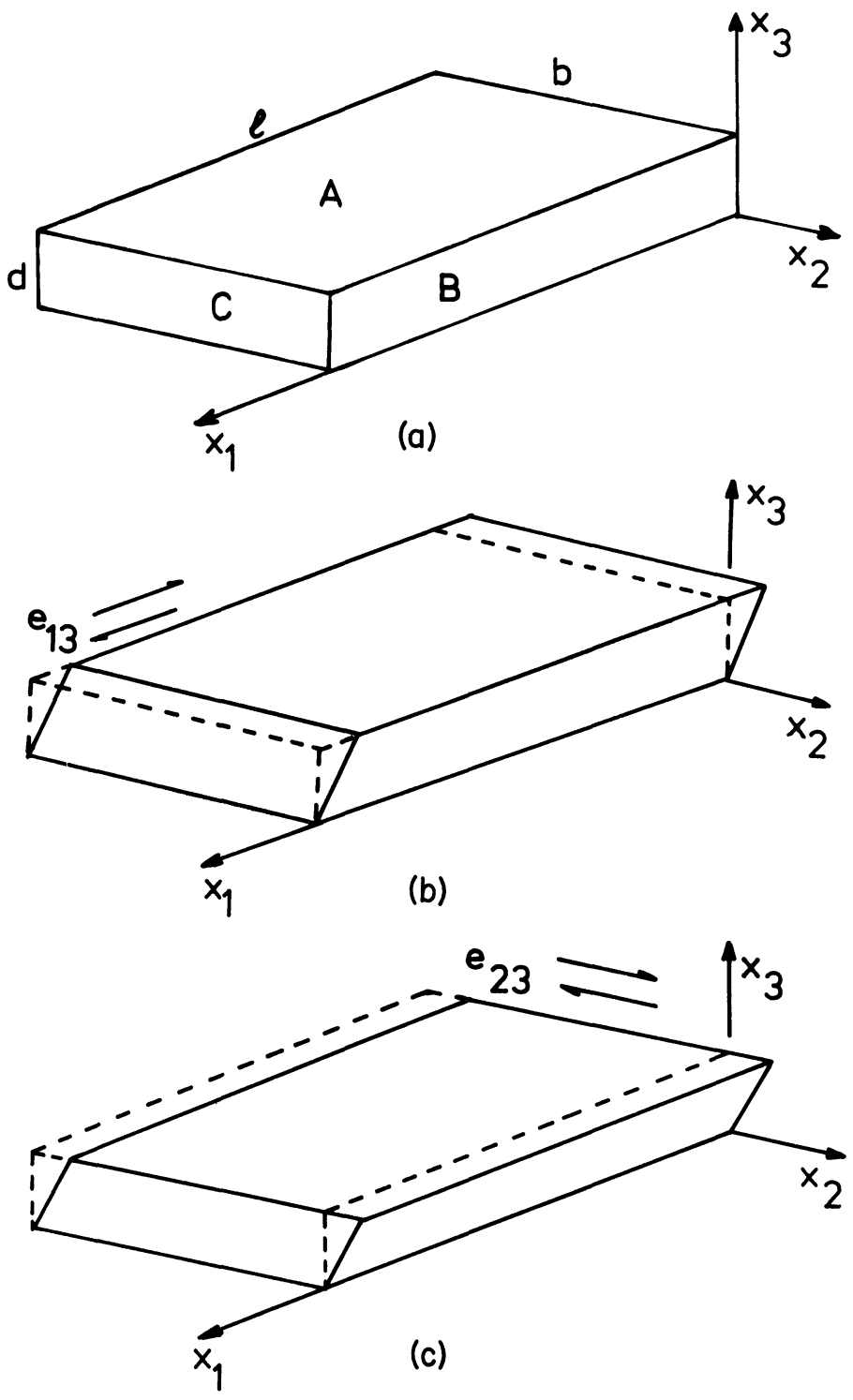

FIGURE 8 (a) Schematic representation of a flat grain in a cold rolled sheet. $x_{1}$ is the rolling direction, $x_{2}$ the transverse direction and $x_{3}$ the sheet plane normal. (b) A simple shear $E_{13}$. (c) A simple shear $E_{23}$. 
metals.

$$
\begin{aligned}
& 0 \leqslant \varphi_{1} \leqslant \pi / 2, \text { step: } \pi / 18 \\
& 0 \leqslant \varphi_{2} \leqslant \pi / 2, \text { step: } \pi / 18 \\
& 0 \leqslant \Phi \leqslant \pi / 2, \text { step: } \pi / 18
\end{aligned}
$$

This means $19^{3}=6859$ nodal points. So the computer programs for the "lath" version of the relaxed Taylor theory must be used to compute these $6859 M$-values. This must be done for all 210 deformation modes, each described by a strain rate tensor given by Eq. (37). No simplifications are possible. So a quite large amount of calculations would be required. For this reason, this model was not implemented.

\subsection{Using the "pancake" model}

5.3.1. Reduction of the number of deformation modes. The method is basically the same as in the "lath" case, but this time an important reduction of the amount of calculations is possible. It is sufficient to use the relaxed Taylor-Bishop-Hill method to produce the $6859 M$-values for 21 deformation modes instead of 210 .

The reason for this is, that the $M(g)$ functions that are calculated for the 21 deformation modes associated to the case $\Psi=0$ can be used to construct the $M(g)$ function associated to other angles $\Psi$ as well.

Let $g$ be the crystal orientation with respect to the reference system of the sample $\left\langle x_{i}\right\rangle$ and let $g^{P}$ represent the orientation of the same crystal with respect to the system $\left\langle x_{i}^{P}\right\rangle$ (Figure 7).

Theorem. The $M$-values of individual crystallites, as calculated by the pancake version of the relaxed Taylor theory, depend on $g^{P}$ and on $\dot{E}_{i j}^{P}$, but not on $\Psi$.

Proof. Let us construct two distinct deformation modes using the same values for $\dot{E}_{i j}^{P}$ but different values for $\Psi$ :

$$
\begin{aligned}
& \Psi=0 \quad \text { (A) } \\
& \Psi \neq 0 \quad \text { (B) (Fig. 7) }
\end{aligned}
$$


In case (A), we would write the equations of the pancake version of the relaxed Taylor theory (Van Houtte, 1982) in the reference system $\left\langle x_{i}\right\rangle$, in case (B) we would write them in the reference system $\left\langle x_{i}^{P}\right\rangle$ as shown in Figure 7.

The formal expressions that these equations have in a reference system $\left\langle x_{i}^{P}\right\rangle$ depend on (Van Houtte, 1982):

- the crystal orientation $g^{P}$;

- the nature of the slip and/or twinning systems, which is the same in case $\mathbf{A}$ and $\mathbf{B}$;

- the expression for the strain rate tensor that describes the imposed strain. It is the same in case (A) and (B) because we have deliberately chosen to use the same values for $\dot{E}_{i j}^{P}$ in both cases.

- the mathematical expression of the components of the strain rate tensor that have been relaxed. In the pancake version of the relaxed Taylor model, $\dot{E}_{13}$ and $\dot{E}_{23}$, expressed in the reference system of the sample, are relaxed.

This means, that the strain rate of an individual crystallite is allowed to differ from the imposed strain rate by a "freedom strain rate tension" given by:

$$
\dot{E}_{i j}^{\prime}=F_{1}\left[\begin{array}{ccc}
0 & 0 & 1 \\
0 & 0 & 0 \\
1 & 0 & 0
\end{array}\right]+F_{2}\left[\begin{array}{ccc}
0 & 0 & 0 \\
0 & 0 & 1 \\
0 & 1 & 0
\end{array}\right]
$$

in which $F_{1}$ and $F_{2}$ are "free", i.e. they are unknowns that are to be calculated, just as the slips. This "freedom expression" can be written in the $\left\langle x_{i}^{P}\right\rangle$ system by using the transformation formulas Eqs. (36-37):

$$
\dot{E}_{i j}^{\prime P}=F_{1}\left[\begin{array}{ccc}
0 & 0 & \cos \Psi \\
0 & 0 & \sin \Psi \\
\cos \Psi & \sin \Psi & 0
\end{array}\right]+F_{2}\left[\begin{array}{ccc}
0 & 0 & -\sin \Psi \\
0 & 0 & \cos \Psi \\
-\sin \Psi & \cos \Psi & 0
\end{array}\right]
$$

Expression (41) is not formally identical to expression (42). But $F_{1}$ and $F_{2}$ are unknown, so they can be replaced by an equivalent set 
of unknowns:

$$
\begin{aligned}
& F_{1}^{P}=F_{1} \cos \Psi-F_{2} \sin \Psi \\
& F_{2}^{P}=F_{1} \sin \Psi+F_{2} \cos \Psi
\end{aligned}
$$

Equation (42) now becomes:

$$
\dot{E}_{i j}^{P}=F_{1}^{P}\left[\begin{array}{lll}
0 & 0 & 1 \\
0 & 0 & 0 \\
1 & 0 & 0
\end{array}\right]+F_{2}^{P}\left[\begin{array}{lll}
0 & 0 & 0 \\
0 & 0 & 1 \\
0 & 1 & 0
\end{array}\right]
$$

which this time is formally identical to Eq. (41).

So the equation of case (A) and case (B) can be brought into formally identical forms. Thus the solutions must be formally identical as well. It can be concluded, that the resuiting $M$-values are the same for case A and B.

Note that this theorem cannot be proved for the "lath" version of the relaxed Taylor theory. In that case, Eq. (41) would be:

$$
\dot{E}_{i j}^{\prime}=F_{1}\left[\begin{array}{ccc}
0 & 0 & 1 \\
0 & 0 & 0 \\
1 & 0 & 0
\end{array}\right]
$$

After transformation to the $\left\langle x_{i}^{P}\right\rangle$ system, this becomes:

$$
\dot{E}_{i j}^{\prime P}=F_{1}\left[\begin{array}{ccc}
0 & 0 & \cos \Psi \\
0 & 0 & \sin \Psi \\
\cos \Psi & \sin \Psi & 0
\end{array}\right]
$$

This is not formally identical to eq. (46) and there are no tricks such as the transformation of unknowns (Eq. (43-44)) to make it identical.

5.3.2. Calculation of 21 sets of $6859 M$-values. The theorem has shown, that for all angles $\Psi$, the function $M\left(g^{P}\right)$ is the same. So the pancake version of the Taylor-Bishop-Hill model was used to calculate the $M\left(g^{P}\right)$-distribution functions for 21 deformation modes, given by Eqs. (24-32) and Eqs. (36-37) in which $\Psi$ was set equal to zero. For each of these 21 deformation modes, 6859 $M$-factors were obtained. A special feature of the computer code was, that the $M$-values which would be found by a full-constraints 
(F.C.) Taylor-Bishop-Hill model were intermediary results of the calculation. So 21 additional distributions of each 6859 F.C. $-M$ values were simultaneously obtained.

Such calculations have been performed for f.c.c. metals, which have twelve potential slip systems $(\{111\}\langle 110\rangle)$, and for b.c.c. metals which have 24 potential slip systems $(\{110\}\langle 111\rangle$ and $\{112\}\langle 111\rangle)$. The critical resolved shear stresses were assumed equal on all potential slip systems.

The series expansions that approximately describe these $M\left(g^{P}\right)$ distributions have been sought. In analogy with Eq. (15):

$$
M\left(g^{P}\right)=\sum_{l}^{L} \sum_{\mu}^{N_{1}(l)} \sum_{v}^{N_{2}(l)}\left(m^{p}\right)_{l}^{\mu v} \dot{T}_{l}^{\mu v}\left(g^{P}\right)
$$

The $\dot{T}_{l}^{\mu v}\left(g^{P}\right)$ are cubic-orthorhombic functions (Bunge, 1982), since strain tensors of the type shown by Eq. (23) have an orthorhombic (class B) symmetry (Van Houtte and Aernoudt, 1976).

The $\left(m^{P}\right)_{l}^{\mu \nu}$ coefficients can be obtained by numerical integration through Euler space:

$$
\left(m^{P}\right)_{l}^{\mu v}=(2 l+1) \oint M\left(g^{P}\right) \dot{\tilde{T}}_{l}^{\mu v}\left(g^{P}\right) d g^{P}
$$

5.3.3. Calculation of the coefficients of $M(g)$ from those of $M\left(g^{P}\right)$. The coefficients $m_{l}^{\mu \nu}$ of the $M(g)$ function can be calculated from the $\left(m^{p}\right)_{l}^{\mu \nu}$ coefficients of the $M\left(g^{p}\right)$-function for $\Psi$-angles that are different from zero (Figure 7).

In a first step, the symmetric $\left(m^{P}\right)_{l}^{\mu \nu}$-coefficients must be desymmetrisized with respect to the "sample symmetry" (Bunge, 1982):

$$
\left(m^{P}\right)_{l}^{\mu \nu} \rightarrow\left(m^{P}\right)_{l}^{\mu n},\left(m^{P}\right)_{l}^{\mu,-n}
$$

This is done by means of the following formulas:

i) for $v=1$ :

$$
\begin{aligned}
n & =0 \\
\left(m^{P}\right)_{l}^{\mu n} & =\left(m^{P}\right)_{l}^{\mu \nu}
\end{aligned}
$$


ii) for $v>1$ :

$$
\begin{gathered}
n=2(v-1) \\
\left(m^{P}\right)_{l}^{\mu n}=\frac{\sqrt{ } 2}{2}\left(m^{P}\right)_{l}^{\mu \nu} \\
\left(m^{P}\right)_{l}^{\mu,-n}=(-1)^{l} \frac{\sqrt{ } 2}{2}\left(m^{P}\right)_{l}^{\mu \nu}
\end{gathered}
$$

The $m_{l}^{\mu n}$ coefficients (unsymmetric with respect to the sample symmetry) of the $M(g)$ distribution (Eq. (15)) can now be calculated:

$$
m_{l}^{\mu n}=\sum_{s=-l}^{l}\left(m^{P}\right)_{l}^{\mu s} T_{l}^{n s}\left(g_{\Psi}\right)
$$

in which $g_{\Psi}$ represents the rotation over $-\Psi$ around $x_{3}$ (Figure 7), i.e. it has the Euler angles:

$$
\begin{aligned}
\varphi_{1} & =-\Psi \\
\Phi & =0 \\
\varphi_{2} & =0
\end{aligned}
$$

$T_{l}^{n s}$ are "unsymmetric" generalized spherical harmonics. They have been calculated once and for all for each of the 10 values of $\Psi$ that are considered.

The coefficients of $M(g)$ must now be made symmetric again:

$$
m_{l}^{\mu n}, m_{l}^{\mu,-n} \rightarrow m_{l}^{\mu v}
$$

In order to be able to treat monoclinic textures (such as torsion textures), a type of $m_{l}^{\mu v}$ coefficients that has a monoclinic sample symmetry must be used. A method of constructing an orthogonal set of monoclinic coefficients and functions has been described by Van Houtte, Aernoudt and Sekine (1981). It is summarized by:

i) for $n=0$ :

$$
\begin{array}{cc}
v=1 & \\
m_{l}^{\prime \mu v}=m_{l}^{\mu n}, m_{l}^{\mu \nu}=0 & \text { for } l=\text { even } \\
m_{l}^{\prime \mu v}=0, m_{l}^{\mu \nu}=m_{l}^{\mu n} & \text { for } l=\text { odd }
\end{array}
$$


ii) for $n>0$

$$
\begin{gathered}
v=1+n / 2 \\
m_{l}^{\prime \mu v}=\frac{\sqrt{ } 2}{2}\left[m_{l}^{\mu n}+(-1)^{l+n} m_{l}^{\mu,-n}\right] \\
m_{l}^{\mu \nu}=\frac{\sqrt{ } 2}{2}\left[m_{l}^{\mu n}-(-1)^{l+n} m_{l}^{\mu,-n}\right]
\end{gathered}
$$

The $m_{l}^{\prime \mu \nu}$ coefficients have an orthorhombic symmetry. The two types together permit a description of a monoclinic distribution function for which $x_{3}$ is a twofold symmetry axis.

So the series expansion of the $M$-values of 210 deformation modes is obtained by calculating $m_{l}^{\prime \mu \nu}$ and $m_{l}^{\prime \mu \nu}$ for the 10 values of $\Psi$ and for the 21 sets of $\left(m^{P}\right)_{l}^{\mu \nu}$ coefficients. These 210 sets of $m$-coefficients are stored once and for all. The only lengthy calculations are those required for the production of the 21 sets of $6859 M$-values (Taylor-type calculations), and, to a lesser extent, the numerical integrations through these sets (Eq. (49)).

5.3.4. Calculation of $\bar{M}$ for a textured sample. Let the texture of the polycrystalline sample be described by $C$-coefficients. The average Taylor factors are then calculated for all 210 deformation modes as shown by Eq. (16). In the case of monoclinic sample symmetry, the explicit form of Eq. (16) is:

$$
\bar{M}=\sum_{l=0}^{L} \sum_{\mu=1}^{N_{1}(l)} \sum_{v=1}^{N_{2}(l)} \frac{m_{l}^{\prime \mu v} C_{l}^{\prime \mu \nu}+m_{l}^{\mu \nu} C_{l}^{\prime \mu v}}{2 l+1}
$$

The definition of $C^{\prime}$ and $C^{\prime \prime}$ for monoclinic sample symmetrices has already been given elsewhere (Van Houtte et al., 1981). In case of orthorhombic sample symmetry (e.g. rolling textures), $C_{l}^{{ }^{\mu \nu}}$ are the usual $C$-coefficients and the $C_{I}^{\prime \mu v}$ are zero.

The computer time required for the evaluation of $210 \bar{M}$-values of Eq. (65) is negligible.

\subsection{Using the "full constraints" model}

The same method is used as for the pancake model (see the previous section). The number of deformation modes for which 
$6859 M$-values must be calculated could in principle be reduced from 21 to 7, because in this case the Taylor-Bishop-Hill equations also remain formally identical when the $\left\langle x_{1}^{P}, x_{2}^{P} x_{3}^{P}\right\rangle$ axes are interchanged amongst each other. But there appeared to be no need to implement this property into the calculation procedure, since the 21 full-constraints $M$-values had already been calculated anyway as intermediary results of the pancake calculations.

\section{GENERATION OF PLANE SECTIONS THROUGH THE YIELD LOCUS}

It has been explained in the previous section (in the case of orthorhombic sample symmetry) how $210 \bar{M}$-values can be obtained for a textured sample. In appendix I it is shown how it can be extended to a total set of $399 \bar{M}$-values. Neglecting crystallographic Bauschinger effects (see section 4.3) permits to adopt the same $\bar{M}$-values for the deformation modes of opposite sign. So the $210 \bar{M}$-values permit to find the equations of 798 hyperplanes. They represent (with a certain angular resolution) the complete set of hyperplanes that are tangent to a three-dimensional section of the fivedimensional yield locus as defined by:

$$
S_{23}=S_{13}=0
$$

(See section 2 and section 4).

This means that all plane sections that respect Eq. (21) can now be made. The procedure goes as follows:

i) the plane section is defined, and its analytical equations are derived.

ii) the latter are used to compute analytically the intersections with the 210 hyperplanes.

The plane section is defined in a user-friendly form. Figure 9 shows the general form of such plane section.

It is a curve plotted in a $(x, y)$ reference system. The user wants the $x$-axis to represent a certain stress component and the $y$-axis another. He merely needs to define the unit vectors $\mathbf{S}_{\mathbf{x}}$ and $\mathbf{S}_{\mathbf{y}}$ in terms of stress components. Let the six dimensions of stress space 


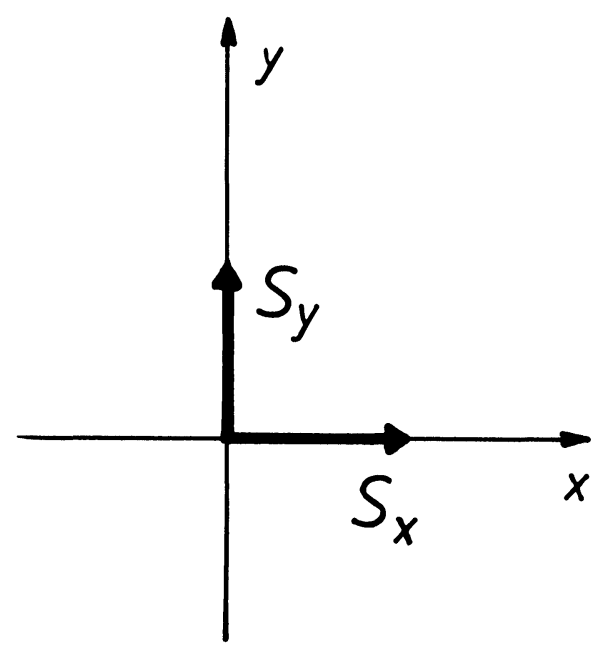

FIGURE $9(x, y)$ is the orthogonal coordinate system of a two dimensional section of stress space. It is defined by specifying the unit vectors $\mathbf{S}_{\mathbf{x}}$ and $\mathbf{S}_{\mathbf{y}}$ in terms of stress components.

respectively correspond to $S_{11}, S_{22}, S_{33}, S_{23}, S_{31}$ and $S_{12}$. Suppose that one wants a $S_{11}, S_{12}$-section.

The two unit vectors must then be specified as follows:

$$
\begin{aligned}
& \mathbf{S}_{\mathbf{x}}=\{1,0,0,0,0,0\} \\
& \mathbf{S}_{\mathbf{y}}=\{0,0,0,0,0,1\}
\end{aligned}
$$

It is clear that in view of condition (21), the $S_{23}$ and $S_{31}$ components must remain zero. It is also possible to ask for two predetermined unit vectors $\mathbf{S}_{\mathbf{x}}$ and $\mathbf{S}_{\mathbf{y}}$ that represent a section by the $\pi$-plane. The vectors $\mathbf{S}_{\mathbf{x}}$ and $\mathbf{S}_{\mathbf{y}}$ can also be specified in terms of stresses that are expressed in a reference system fixed to a tensile test specimen cut from the sheet at an angle $\alpha$ from the rolling direction (Figure 1). Eq. (18) can be written as follows (regarding Eq. (21)):

$$
P_{11} S_{11}+P_{22} S_{22}+P_{33} S_{33}+P_{12} S_{12}=\tau^{\mathrm{c}} \bar{M}
$$

The $P_{i j}$ values that correspond to the 798 hyperplanes are stored once and for all.

The method of finding their intersections with the plane section 
defined by $\mathbf{S}_{\mathbf{x}}$ and $\mathbf{S}_{\mathbf{y}}$ consists in expressing $P_{i j}$ in a different reference system (in stress space) for which $\mathbf{S}_{\mathbf{x}}$ or $\mathbf{S}_{\mathbf{y}}$ belong to the set of basis vectors. It then becomes very easy to find the intersection. The method has been described in detail elsewhere (Mols, 1982).

The computer code that draws the yield surface section also makes a printout of the strain tensors normal to the yield curve, including those strain components that are invisible in the plane section itself.

\section{EXAMPLES OF RESULTS}

A few examples of results both for f.c.c. metals and b.c.c. metals will be given here.

The $M$-values used for f.c.c. metals have been calculated assuming $\{111\}\langle 110\rangle$ slip systems. For b.c.c. metals, the existence of 24 slip systems was assumed $(\{110\}\langle 111\rangle$ and $\{112\}\langle 111\rangle)$, with equal critical resolved shear stresses on all systems. The resulting $M$ values are somewhat smaller than those obtained for f.c.c. metals.

Figure 10a shows the $\pi$-plane section for an isotropic f.c.c. metal, according to the full constraints model. The result of the relaxed constraints (pancake) model is given by Figure $10 \mathrm{~b}$.

The yield curve of Figure 10a looks like a Tresca yield curve (hexagon) with rounded corners. Compared to this, the stress levels of the "pancake" curve (Figure 10b) are ca. 10\% smaller. It is interesting to note, that for $S_{22}=S_{33}=0$, the radius of curvature is very small, suggesting a vertex.

Figure 11a shows the $\pi$-plane section for an isotropic b.c.c. metal (F.C. Method). This curve is much more rounded off than the f.c.c. curve of Figure 10a; it is closer to the von Mises circle than to the Tresca hexagon. Figure 11b shows the same according to the relaxed constraints (pancake) model. Again, the stress levels are reduced; a region of small radius of curvature is observed near the points $S_{22}=S_{33}=0$, but not to the extent of suggesting a vertex.

Figure 12a shows the $S_{11}-S_{22}$ section of a b.c.c. metal, namely a steel sheet of industrial quality. Incomplete $\{110\},\{200\},\{112\}$ and $\{301\}$ pole figures were measured and treated by a computer in order to obtain the even $C_{l}^{\mu \nu}$ coefficients after which the odd coefficients were estimated as well. The methods have been 


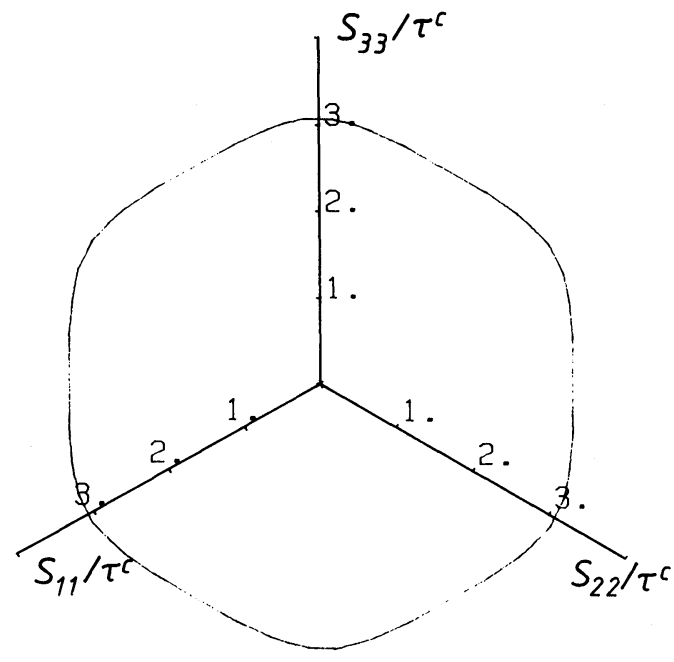

(111) $<110>$ SLIP

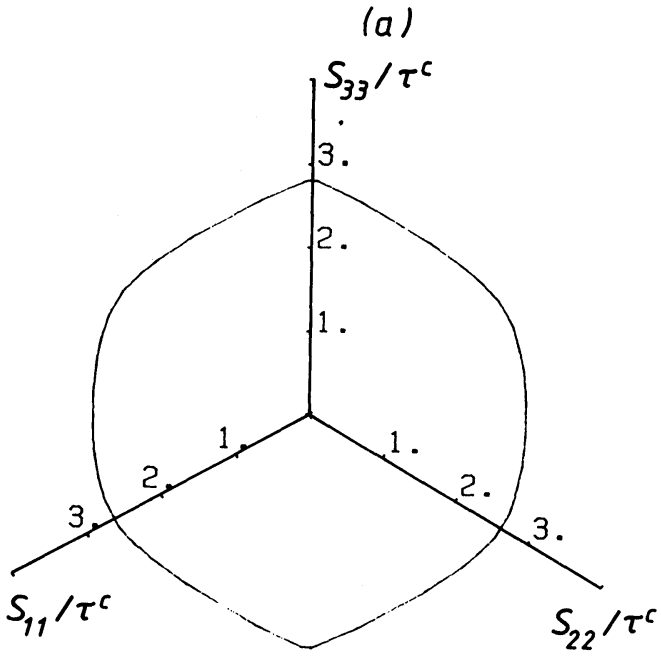

$(111)<110>$ SLIP R.C. (PANCAKE)

(b)

FIGURE $10 \pi$-plane section of the yield surface of a texture-free f.c.c. polycrystal. (a) according to the F.C. model. (b) according to the R.C. (pancake) model. 




(a)

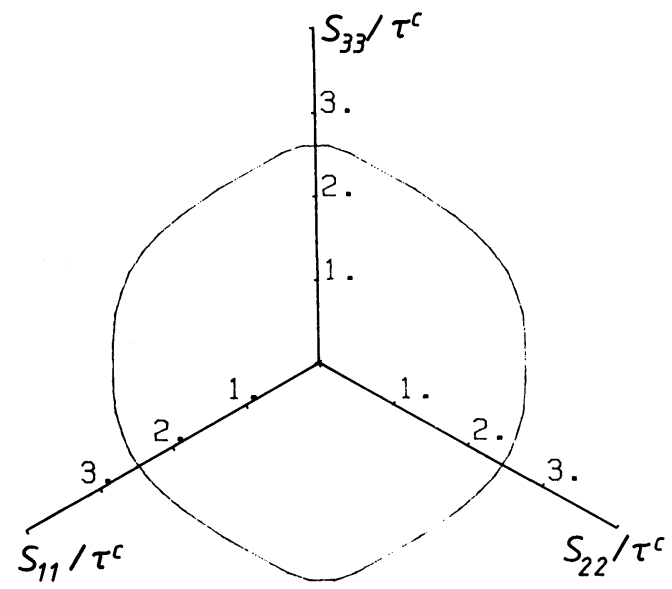

$(111)+(112)<110\rangle$ SLIP R.C. (PANCAKE)

(b)

FIGURE $11 \pi$-plane section of the yield locus of a texture-free b.c.c. polycrystal. (a) according to the F.C. model. (b) according to the R.C. (pancake) model. 


described elsewhere (Van Houtte, 1983 and 1984a). The fullconstraints method was used for the curves of Figure 12a, while the pancake method was used for Figure 12b. In both cases the $\alpha=0^{\circ}$ and the $\alpha=45^{\circ}$ curves are given (see Figure 1).

According to these calculations, there should be in-plane shearing when performing uniaxial tensile tests at $45^{\circ}$. The values of the $\dot{E}_{12} / \dot{E}_{11}$ ratios were taken from the printouts that accompany the curves of Figure 12a-b. At $S_{22}=0$, a value of -0.143 was found for the full-constraints case, and a value of 0.143 for the pancake case, suggesting a quite different strain anisotropy. Exactly which model applies best is the object of an experimental investigation under course. However, the curves of Figure 12a and Figure 12b predict the same $R$-values ( 2.45 for $\alpha=0^{\circ}$ and 1.67 for $\alpha=45^{\circ}$ ) for the full constraint and the relaxed cases.

The method can of course also be applied to a theoretically predicted deformation texture. Figure $13 a-b$ show an example of this. A b.c.c. rolling texture was simulated using the pancake version of the relaxed Taylor theory, extended with Wintenberger's second-order selection criterion for those cases where the solution for the slip are not unique (Renouard and Wintenberger, 1981). A uniform texture consisting of 147 separate crystallites was chosen as starting texture. Then 50 increments of $1 \%$ thickness reduction (plane strain) were simulated, followed by 30 increments of $5 \%$ strain each. A total true strain $\epsilon=2$ was so obtained, representing a thickness reduction of $86 \%$. A continuous O.D.F. was derived from the 147 discrete orientations in the following way:

- For each orientation, a Gaussian distribution was considered:

$$
f(g)=S_{0}^{-\Phi^{2} / \Phi^{2}} \mathrm{e}
$$

$\Phi$ is the minimal rotation angle, required to rotate the considered crystallite to the orientation $g$.

$\Phi_{0}$ is the spread of the Gauss distribution. Its value was $7^{\circ} . S_{0}$ is chosen in order to normalize the texture function:

$$
\oint f(g) d g=1
$$

- The O.D.F. of the texture as a whole is the weighted average of the 147 texture functions given by Eq. (69). It is quite easy to 

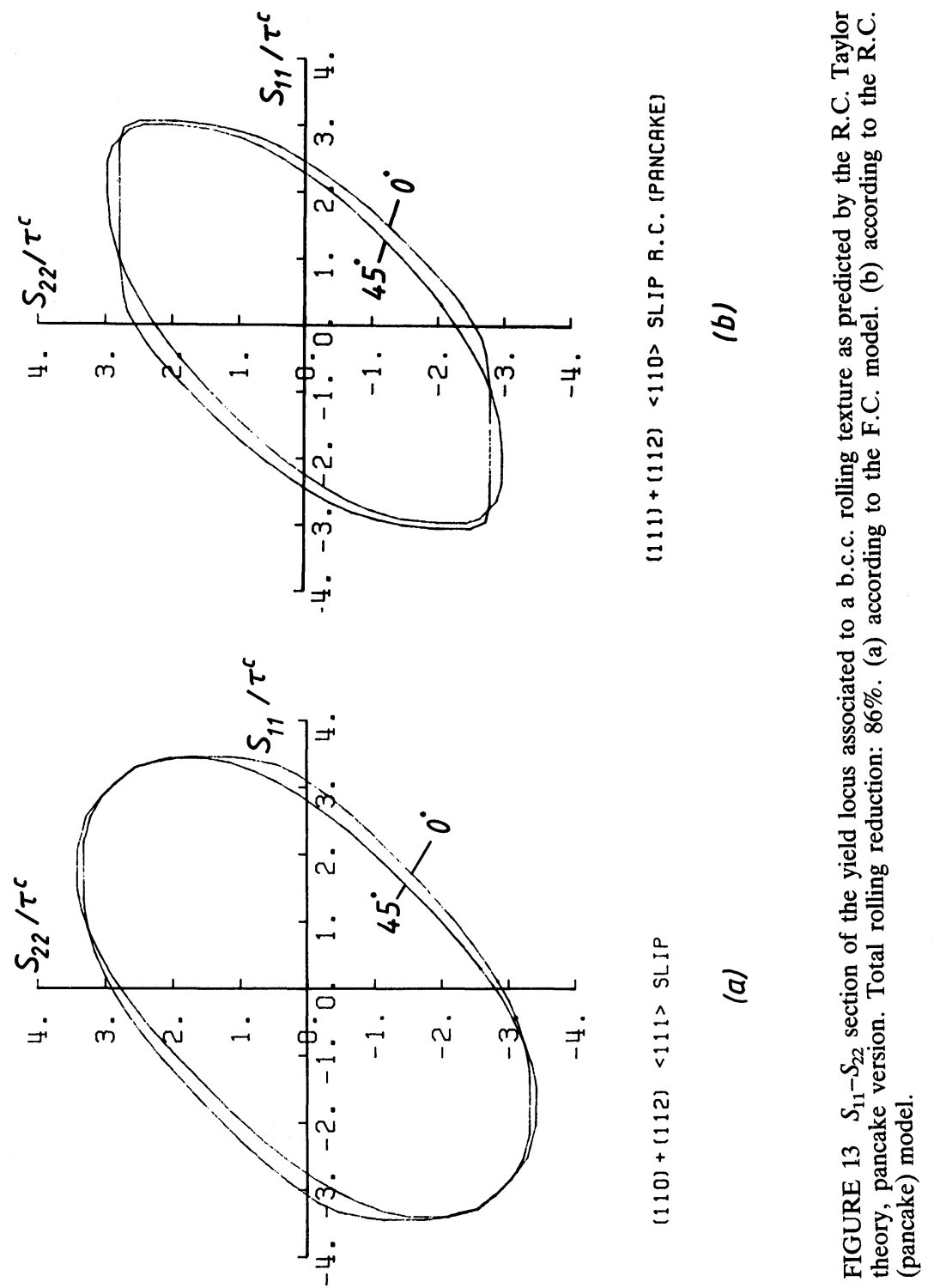
calculate both the even and the odd $C_{l}^{\mu \nu}$-coefficients of such texture (Bunge, 1982). These coefficients were used to calculate the yield locus. Figure 13a shows a $S_{11}-S_{22}$ section (both for $\alpha=0^{\circ}$ and $\alpha=45^{\circ}$ ), using the "full constraint" $M$-values" (although the simulated texture was produced by the relaxed constraints method). The printout of the strain tensors provided the following results:

- $R$-values: 2.45 for $\alpha=0^{\circ}$ and 2.31 for $\alpha=45^{\circ}$

- $\dot{E}_{12} / \dot{E}_{11}=-0.15$ for $\alpha=45^{\circ}$

Figure 13b shows the $S_{11}-S_{12}$ section of the yield loci calculated using the "pancake" $M$-values.

- $R$-values: 1.7 for $\alpha=0^{\circ}$ and 2.4 for $\alpha=45^{\circ}$

- $\dot{E}_{12} / \dot{E}_{11}=0.15$ for $\alpha=45^{\circ}$

Figure 14a shows the $S_{22}-S_{12}$ section of the yield locus of the surface of a solid copper bar after torsion testing. The total shear at the surface was equal to 5 . The $\{100\}$ and $\{111\}$ pole figures are given by Figure 4; the O.D.F. has been reported earlier (Van Houtte et al., 1981). Figure 3 gives the conventions for the reference axes. The component $S_{12}$ is the shear stress induced by the torsional deformation. $S_{22}$ is the normal stress in axial direction. Suppose a free end torsion test. At first sight, one would think that all stress components are zero except $S_{12}$. This stress is represented by $A$ in Fig. 14a. The normal to the yield locus is not perpendicular to the $S_{22}$ axis. This means that in addition to the shearing, there should be some extension of the sample. However, the printout of the strain tensors reveals that this is not an axisymmetric elongation, but a plane strain elongation, the strain in radial direction being zero.

Such deformation would be possible if the sample was a thinwalled tube. In addition to the torsional shearing, the tube would become longer; its radius would become smaller, while the wall thickness would remain constant. The rate of change of the radius would equal minus the rate of change of the length. This has also been found by Canova et al. (1985), who used a theoretically predicted torsion texture in order to derive the yield surface.

The elongation of a solid bar must however be axisymmetric. This means that the rate of change of its radius must be equal to minus half the rate of length change. This would require the building up of 


a positive $S_{11}$ (tangential) normal stress, while probably also inducing axial stresses $S_{22}$. The free end indeed does not require that $S_{22}$ is zero at the surface, only that the average of the $S_{22}$ component over the entire cross section must be zero.

An exact solution of the problem of predicting the ratio of shearing/elongation of a solid bar during a torsion test would be quite complex. The stress components $S_{11}, S_{22}, S_{33}$ and $S_{12}$ are all expected to vary strongly from the surface to the centre. The stress equilibrium equations should be taken into account. Moreover, the texture and the degree of work hardening also vary from the surface to the centre.

An approximate solution that only accounts for the outside layer is possible. One way of doing it would be to transform the measured O.D.F. into a set of discrete crystal orientations. Then a procedure which has been proposed by Gil Sevillano, Van Houtte and Aernoudt (1975) can be carried out. A Taylor-Bishop-Hill computer program is used to provide average $M$-values for different strain modes, defined by:

$$
\begin{aligned}
& \dot{E}_{22}=2 \dot{E}_{11}=-2 \dot{E}_{33}=2 \eta \dot{E}_{12} \\
& \dot{E}_{23}=\dot{E}_{31}=0
\end{aligned}
$$

$\eta$ is the ratio between the axial elongation rate and the shearing rate. The average $M$-value must then be plotted as a function of $\eta$. The most probable value of $\eta$ is the one that corresponds to the minimum of $\bar{M}$.

The macroscopic stress tensor would then be obtained as the average of the Bishop-Hill stress tensor of the individual crystallites, at least when the theorem holds that the macroscopic stress of a polycrystalline aggregate equals the average of the microscopic stresses of the individual crystallites, as has been suggested by Canova et al. (1985).

Another method, which avoids the transformation of the $C$ coefficients of the O.D.F. into a discrete set of crystallite orientations, will be explained below. It is based on a thorough exploration of the yield surface. The basic idea is to look for stress states for which the strain rate tensors (given by the normals to the yield locus) are of the type given by Eqs. (71-72).

Because of the theorem described in section 2, Eq. (72) implies 
that

$$
S_{23}=S_{31}=0
$$

so that the method described in the previous sections is sufficient to construct the yield surface. A set of plane sections of the following type is made:

- Eq. (73);

$$
\begin{gathered}
S_{33}=0 \\
S_{11} / S_{22}=\operatorname{tg} \rho
\end{gathered}
$$

$\rho$ is given values between $-90^{\circ}$ and $90^{\circ}$ in steps of $15^{\circ}$. Figure $14 \mathrm{~b}$ gives an example of such section. They are implemented by the following choices of the unit vectors $S_{x}$ and $S_{y}$, defined in section 6 (Figure 9):

$$
\begin{aligned}
& \tau^{\mathfrak{c}} \cdot \mathbf{S}_{\mathbf{x}}=\{\sin \rho, \cos \rho, 0,0,0,0\} \\
& \tau^{\mathfrak{c}} \cdot \mathbf{S}_{\mathbf{y}}=\{0,0,0,0,0,1\}
\end{aligned}
$$

Let $x$ and $y$ be the coordinates of the points of the resulting yield curve (Figure 9). It is seen that, besides Eqs. (73-74),

$$
\begin{aligned}
& S_{12}=\tau^{c} y \\
& S_{11}=\tau^{c} x \sin \rho \\
& S_{22}=\tau^{c} y \cos \rho
\end{aligned}
$$

It was intended, by examining the printouts of the strain rate tensors, to look for stress states for which the strain rate tensor represents a superposition of a torsion and an axisymmetric elongation (see Eqs. (71-72)). The associated plastic work would then be calculated:

$$
\dot{W}=S_{i j} \dot{E}_{i j}
$$

This plastic work must be produced by the external torque that is applied to the test sample. The twist angle that is realized is proportional to the shear $E_{12}$. So the "resistance" of the material against twisting is proportional to the quantity $\dot{W} / \dot{E}_{12}$. Of all stress states for which the strain rate satisfies Eqs. (71-72) (for example, the stress state $B$ in Figure 14b), the one that minimizes this quantity is supposed to be the most probable. 
Solutions were found in the sections $\rho=0^{\circ}, 15^{\circ}, 30^{\circ}$ and $45^{\circ}$. For more information, an additional section at $\rho=37.5^{\circ}$ was produced. The minimum for $\dot{W} / \dot{E}_{12}$ was found for $\rho=30^{\circ}$ and $37.5^{\circ}$ and for a positive value of $\eta$, the ratio of the axial elongation to the macroscopic shear. $\dagger$ Figure 15 shows the evolution of $\dot{W} / \dot{E}_{12}$ as a function of $\rho$. For reasons of clarity, only the solutions for which $\eta$ was positive are shown. The figure also shows the associated values of $\eta, S_{12}, S_{11}$ and $S_{22}\left(S_{33}\right.$ being zero).

In the vicinity of the minimum, the values of $\dot{W} / \dot{E}_{12}$ and $\eta$ were found to be quite intensitive to $\rho$. The ratio between $S_{11}$ and $S_{22}$ is of course sensitive to $\rho$, much less however their average.

According to these calculations, the rate of elongation $\eta$ should be no less than $11.5 \%$. The stress state consists of a shear stress (as expected in a torsion test) combined with a far from negligible biaxial tensile stress. The elongation rate $\eta$ is probably overestimated when compared to experimental results, the main reason being the neglection of the role of the inner layers of the sample, which probably have a smaller tendency for elongation than the surface layer. It can also be expected that the hydrostatic component of the stress would decrease from the surface to the centre of the sample, thus reducing the stresses $S_{11}$ and $S_{22}$ and provoking a compressive stress in radial direction.

Figure 16 shows true yield curves (obtained by the present method) and some "principal strain yield loci" obtained for a sheet with a very sharp texture: a Gaussian distribution with a narrow spread $\left(\Phi_{0}=7^{\circ}\right.$, Eq. (69)). Its centre of gravity lies exactly on the (110) [001] (Goss) orientation. The F.C. $M$-factors for b.c.c. metals were used. The yield curves have been calculated for $\alpha=22.5^{\circ}$ and $\alpha=45^{\circ}$ (Figure 1). It is seen that the differences between the true yield curve and the principal strain yield curve are quite spectacular. The same holds for the $R$-values: for example for $\alpha=45^{\circ}$, the "principal strain" yield curve predicts a value of 0.786 and the "true" yield curve a value of 0.287 .

A similar comparison carried out for the experimental O.D.F. of Figure 12a however only led to a very small difference between the "true" yield curve and the principal strain yield curve. At present it

$\dagger$ This means, that the sample should elongate when a torsion test would be carried out in the same sense as the prestraining. 
66

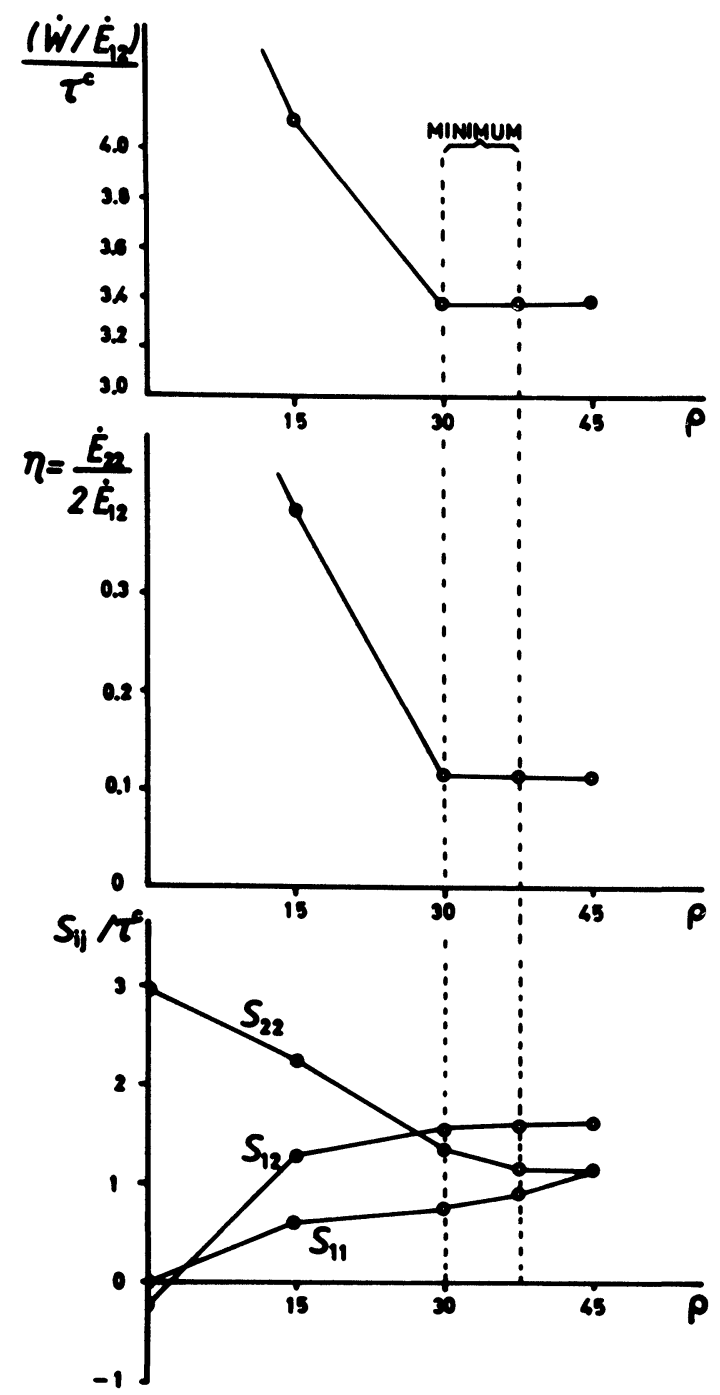

FIGURE 15 Analysis of the plastic anisotropy near the surface of a copper test sample after a torsional deformation of $500 \%$. In different plane sections of the yield locus, characterised by $\rho$ (Eqs. 74-75), stress states are sought for which the corresponding strain mode is a torsional deformation $\left(\dot{E}_{12}\right)$ combined with an axisymmetric elongation or compression $\left(\dot{E}_{22}\right)$. The figure shows these stress states. The minimum for $\dot{W} / \dot{E}_{12}$ gives the most probable solution for $\rho$. Due to lack of resolution of the method, it was impossible to differentiate between $\rho=30^{\circ}$ and $\rho=37.5^{\circ}$. The corresponding values of the rate of elongation $\eta$, the torsional stress $S_{12}$, the axial stress $S_{22}$ and the normal stress in tangential direction $S_{11}$ are also shown. 




FIGURE 16 "True" yield curves and "principal strain" yield curves for $\alpha=22.5^{\circ}$ and $\alpha=45^{\circ}$, for a sheet of a b.c.c. metal with a very strong (110)[001] texture.

is not known whether this holds for all textures of polycrystalline samples that are of industrial interest. More experience must be gained before a definitive conclusion can be made.

\section{CONCLUDING REMARKS}

In regard of analysis of yield loci, it is a tremendous advantage to know the crystallite orientation distribution function of a textured sample in the form of a set of $C_{l}^{\mu \nu}$-coefficients of a series expansion, either obtained from pole figure measurements on real samples or from Taylor-type deformation texture predictions. The use of these coefficients permit high-speed calculations of average Taylor factors, which can then be used to define hyperplanes in stress space that are tangent to the yield locus. Mols, Van Praet and Van Houtte (1984) used this method for an approximate construction of the complete yield locus, i.e. in full five-dimensional stress space. 
An additional computer program permitted to produce at wish plane sections through it. This method used 51408 hyperplanes. Although the calculations were not unreasonably lengthy, an interactive exploitation proved to be too expensive for a university laboratory. A truly low-cost procedure is proposed in the present paper. It can be applied to textures for which the $x_{3}$-axis is a two-fold "sample" symmetry axis. The complete yield locus is no longer obtained, but only its section by the $\left(S_{11}-S_{22}-S_{33}-S_{12}\right)$ subspace. It however is complete in that subspace. Although the method is less general than its predecessor, it nearly covers all practical applications.

The average $M$-factors needed for the hyperplanes are first calculated. In a next step, the user defines the plane sections that he wants. "True" sections are obtained: when e.g. a $\left(S_{11}-S_{21}\right)$ section is produced, then the shear stress $S_{12}$ is truly zero, and the shear rate $\dot{E}_{12}$ is computed together with the shear rates $\dot{E}_{11}$ and $\dot{E}_{22}$. The values of such predicted shears (produced by the older method) have been found to correlate positively with experimentally observed shearing of tensile samples cut from textured sheets (Mols et al., 1984).

The $M$-value data bases used in the present model for the first time not only include the usual $M$-values of f.c.c. metals $(\{111\}\langle 110\rangle$ slip) but also those of b.c.c. metals $(\{110\}$ and $\{112\}$ slip planes, $\langle 111\rangle$ slip directions). In this regard, there is no need for approximate solutions such as those offered by the "pencil glide" model.

Not only the full constraints Taylor-Bishop-Hill model was used, but also the "pancake" version of the relaxed Taylor model (Van Houtte, 1982). This model is thought to be suited for rolled metal sheets which have very flat grains. Work to verify this experimentally is in progress.

The examples given above show, that the method can be used to study the plastic response of anisotropic materials. The experience gained so far suggests, that

i) the yield curves themselves are found with a reasonable precision.

ii) the accuracy of the directions of the normals to the yield curves, i.e. the strain modes, suffer from the relatively limited 
number of hyperplanes used for the analysis (angular resolution ca. $10^{\circ}$ ). It is useless to apply smoothing procedures to the yield curves in the plane sections since we want to know all components of the vectors normal to them, i.e. not only the 2 components that are seen in the plane section, but the 2 other "invisible" components that also exist in the $\left(S_{11}-S_{22}-S_{33}-S_{12}\right)$ subspace as well. A sound multidimensional interpolation procedure is needed. Such procedure has in fact been developed and will be reported in the near future.

When comparing this method to older "principal strain" methods based on series expansions, it can be concluded that:

- the new method perhaps needs more computer time, but this is not a decisive factor since the required time is quite small anyway (except for the calculation of the $M$-value data bank, which has to be performed only once);

- For very sharp (artificial) textures, large differences were found between "true" yield curves produced by the new method and "principal strain yield loci" for $\alpha$ angles other than $0^{\circ}$ or $90^{\circ}$ (Figure 1). Until now, an important difference of this kind was not found for polycrystalline samples of industrial interest. Nevertheless, even in such cases, in-plane shears $E_{12}$ of the order of $10 \%$ of the elongation strain were quite often found by the new method. Needless to say that the "principal strain" methods are unable to calculate these shears.

It is finally repeated here that in this paper, no attempt was undertaken to replace the (FC or RC) Taylor-Bishop-Hill model by an elasto-plastic model. This means that only large offset yield loci (a few percent plastic strain) can be calculated, since it is implicitly assumed that the multislip stage has been reached in all grains.

In future it could be attempted to combine the method presented here and which essentially provides in a means for exploring the whole $S_{11}-S_{22}-S_{33}-S_{12}$ subspace, with a more sophisticated model for polycrystal plasticity (such as an elasto-plastic self-consistent model). It would then be possible to predict small offset yield loci (within the elasto-plastic transition range) as well. 


\section{Acknowledgments}

The author wishes to thank Prof. E. Aernoudt and Mr. K. Mols for their many interesting discussions on the subject, and the NV Sidmar for supporting this research work.

\section{References}

Berveiller, M. and Zaoui, A., J. Engng. Mater. Technol. 106, 295-298 (1984).

Bunge, H. J., Kristall und Technik 5, 145-175 (1970).

Bunge, H. J., Z. Metallkunde 70, 411-418 (1979).

Bunge, H. J., Texture Analysis in Material Science, Butterworths, London (1982).

Bunge, H. J., Schulze, M. and Grzesik, D., Peine/Salzgitter Berichte, Sonderheft (1980).

Canova, G. R., Kocks, U. F., Tomé, C. N. and Jonas, J. J., J. Mech. Phys. Solids 33, 371-397 (1985).

Esling, C., Bunge, H. J. and Muller, J., J. Physique-LETTRES 41, L543-L545 (1980).

Gil Sevillano, J., Van Houtte, P. and Aernoudt, E., Z. Metallkde 66, 367-373 (1975).

Gil Sevillano, J., Van Houtte, P. and Aernoudt, E., Progr. Mater. Sci. 25, 69-412 (1980).

Grumbach, M., Parnière, P., Roesch, L. and Sauzay, C., Mém. Sci. Rev. Metallurgie 72, 241-253 (1975).

Kumar, A. and Hutchinson, W. B., 3e Colloque Europeen sur les Textures de Déformation et de Récristallisation des Métaux et leurs Applications Industrielles, pp. 527-541, Pont-à-Mousson (1973).

Mols, K., Experimentele Kontrole van Berekende Vloeioppervlakken, Engng. Thesis, Department of Metallurgy and Materials Science, Katholieke Universiteit Leuven (1982).

Mols, K., Van Praet, K. and Van Houtte, P., Proc. Seventh International Conference on Textures of Materials, pp. 651-656, C. M. Brakman, P. Jongenburger and E. J. Mittemeijer, eds., Netherlands Society for Materials Science, Zwijndrecht, The Netherlands (1984).

Parnière, P. and Roesch, L., Mém. Sci. Rev. Métallurgie 72, 221-240 (1975).

Renouard, M. and Wintenberger, M., C.r. Acad. Sci. Paris B292, 385-388 (1981).

Sowerby, R., Da Viana, C. S. and Davies, G. J., Mat. Sci. Eng. 46, 23-51 (1980).

Van Houtte, P., Mater. Sci. Eng. 55, 69-77 (1982).

Van Houtte, P., Textures and Microstructures, 6, 1-20 (1983).

Van Houtte, P., Textures and Microstructures, 6, 137-162 (1984a).

Van Houtte, P., Proc. Seventh International Conference on Textures of Materials, pp. 7-23, C. M. Brakman, P. Jongenburger and E. J. Mittemeijer, eds., Netherlands Society for Materials Science, Zwijndrecht, The Netherlands (1984b).

Van Houtte, P. and Aernoudt, E., Mater. Sci. Eng. 23, 11-22 (1976).

Van Houtte, P. Aernoudt, E. and Sekine, K., Proc. Sixth International Conference on Textures of Materials, pp. 337-346, The Iron and Steel Institute of Japan Tokyo (1981). 
APPENDIX I

Reduction of the amount of $M$-value calculations in case of orthorhombic sample symmetry

In the case of orthorhombic sample symmetry, $x_{1}$ also is a twofold symmetry axis of the sample. The stress states associated to the
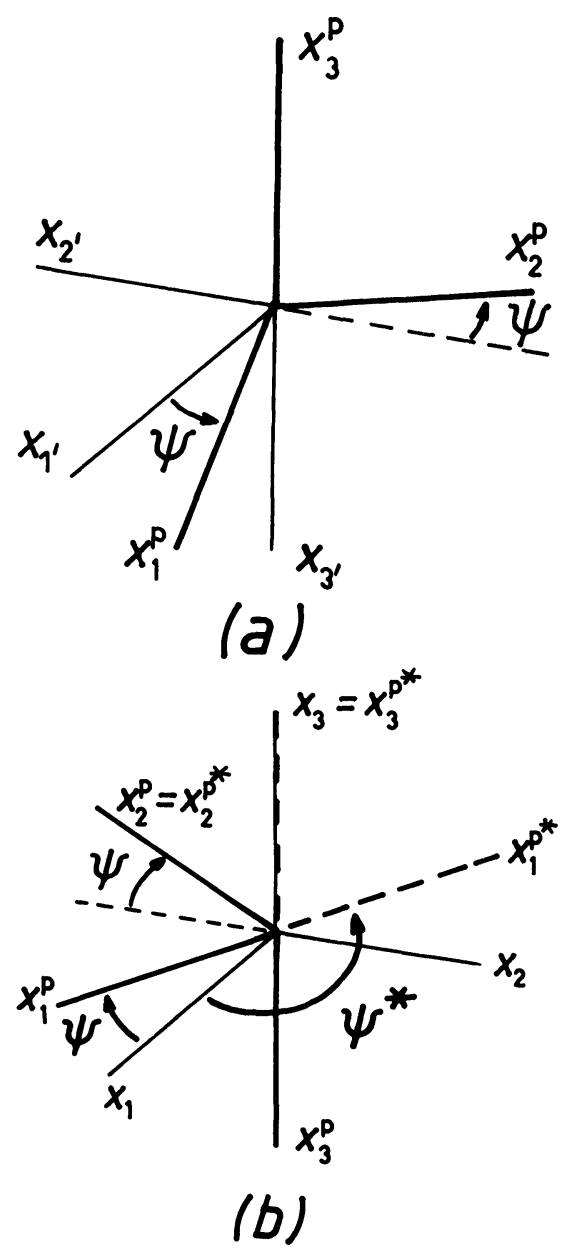

FIGURE $17 x_{1}$ and $x_{3}$ are twofold symmetry axes of the sample (a) the loading axes are conserved, the sample is rotated around $x_{1}$ into its symmetrical position. (b) the configuration of (a) is rotated back as a whole. 
range $\frac{\pi}{2}<\Psi \leqslant \pi$ can then be derived easily from those of the range $0 \leqslant \Psi \leqslant \pi$.

Consider a sample that undergoes a strain characterised by $\Psi$ and $\dot{E}_{11}, \dot{E}_{22}, \dot{E}_{33}$ (Eq. (23), Figure 7). Because of the symmetry, the plastic response of this sample ( $M$-factor) must be the same as that of another sample, derived from the first one by a rotation $\pi$ around $x_{1}$ (while conserving the axes of loading $\left\langle x_{1}^{P}, x_{2}^{P}, x_{3}^{P}\right\rangle$. This rotation brings the sample axes into the positions $\left\langle x_{1}^{\prime}, x_{2}^{\prime}, x_{3}^{\prime}\right\rangle$ (Figure 17a).

Now we rotate the second sample together with its loading system again by an angle $\pi$ around $x_{1}$. The axes $\left\langle x_{1}^{\prime}, x_{2}^{\prime}, x_{3}^{\prime}\right\rangle$ now coincide again with $\left\langle x_{1}, x_{2}, x_{3}\right\rangle$ (Figure $17 \mathrm{~b}$ ), but the axes $\left\langle x_{1}^{P}, x_{2}^{P}, x_{3}^{P}\right\rangle$ do not coincide any more with those of the first sample.

Strain rate tensors or stress tensors are however the same when expressed to reference system $\left\langle x_{i}^{P}\right\rangle$ or $\left\langle x_{i}^{P *}\right\rangle$ (Figure 17b).

In the configuration of Figure 17b, the strain rate tensors can be described by parameters $\Psi^{*}, \dot{E}_{11}^{P *}, \dot{E}_{22}^{P *}, \dot{E}_{33}^{P *}$ which obey to the conventions of Figure 7 and are given by:

$$
\begin{aligned}
\Psi^{*} & =\pi-\Psi \\
\dot{E}_{11}^{P *} & =\dot{E}_{11}^{P} \\
\dot{E}_{22}^{P *} & =\dot{E}_{22}^{P} \\
\dot{E}_{33}^{P *} & =\dot{E}_{33}^{P}
\end{aligned}
$$

This finally means, that the $\bar{M}$-factor associated to the parameters $\Psi^{*}, \dot{E}_{11}^{P *}, \dot{E}_{22}^{P *}, \dot{E}_{33}^{P *}$ (Eqs. $\left.82-85\right)$ is the same as the $\bar{M}$-factor associated to the strain mode described by $\Psi, \dot{E}_{11}^{P}, \dot{E}_{22}^{P}, \dot{E}_{33}^{P}$. 